\title{
A sideways glance. Do you remember your grandmother's food? How epigenetic changes transmit consequences of nutritional exposure from one generation to the next
}

\author{
Maria Laura Scarino
}

Published online: 15 March 2008

(C) Springer-Verlag 2008

The word "epigenetics" was coined by the developmental biologist Conrad H. Waddington in 1942, to indicate the interactions between environment and genetic inheritance leading to the unfolding of alternative phenotypes during embryogenesis and development [11].

Today epigenetics (or epigenomics) mainly refers to the study of heritable structural modifications of genes and chromatin organization, which affect gene expression without modifying primary DNA sequence. Epigenetic processes stabilize the expression profiles in different tissues and are thus essential for development and maintenance of cell type diversity throughout life. Epigenetics encompasses modifications in gene sequence accessibility with the interplay of mechanisms such as DNA methylation, covalent and non-covalent modification of histones and expression of non-coding RNAs, eventually leading to differential expression or silencing patterns in the genome (reviewed in [9]). Chromatin structure and DNA methylation of the genome as a whole is termed the epigenome and varies between different cell types. The epigenome is duplicated along with the genome during cell division but it can also undergo changes during cell differentiation to allow new patterns of gene expression. However, the key question, i.e. how the epigenome is faithfully inherited through cell divisions, has not yet been fully clarified [7].

Changes in the epigenome can be triggered during prenatal life by a variety of environmental factors affecting the mother (nutrition, stress, xenobiotics, nursing behavior) and also, to a minor extent, the father (reviewed in [2]).

M. L. Scarino ( $\square)$

National Research Institute on Food and Nutrition,

Rome, Italy

e-mail: scarino@inran.it
Analysis of the epigenome of monozygotic twins revealed that DNA methylation and histone acetylation patterns diverge as they get older, demonstrating that the same genetic information can be differentially modulated during adult life and offering an epigenetic explanation for phenotypic discordance observed in identical twins [3].

The epigenome is mainly established during fetal development and in this period it can be influenced by maternal nutritional imbalance that leads to perturbation of those processes that have evolved to predict and adjust the phenotype to an expected environment, a mechanism known as developmental plasticity. Later in life this can lead to increased risk of developing metabolic syndrome and cardiovascular disease, among others [4]. Under this perspective, the epigenome can also be described as the "cellular memory" of the nutritional exposure of our mothers or even our grandmothers.

This issue of "sideways glance" highlights some publications produced in 2007 by the "Southampton group". This British research group, investigating how pre-natal dietary restriction can affect phenotype in rats and humans, has recently proposed the "mismatch concept" to explain the developmental origins of good or bad health during life. In the paper by Burdge et al. [1] pregnant rats $\left(\mathrm{F}_{0}\right)$ were fed a protein restricted diet ( $\mathrm{PR}$ ) containing, $50 \%$ of the protein content of a reference diet (RD). The $F_{1}$ and $F_{2}$ females were fed a standard diet, mated to normal males and the liver of $F_{1}$ and $F_{2}$ males was analyzed for methylation state of the glucocorticoid receptor $\left(\mathrm{GR} 1_{10}\right)$ and of the peroxisome proliferator-activated receptor alpha (PPAR $\alpha$ ) genes by methylation sensitive PCR. Expression of $\mathrm{GR}_{10}$ and $\operatorname{PPAR} \alpha$, as well as that of their target genes acyl-CoA oxydase (AOX) and phosphoenolpyruvate carboxykinase (PEPCK), were analyzed by RT-PCR. Methylation state of both the GR $1_{10}$ and PPAR $\alpha$ promoters was significantly 
lower and the expression of AOX and PEPCK was higher, yet not reaching significance for the latter gene, in rats of the PRD groups $\left(F_{1}\right.$ and $\left.F_{2}\right)$. These results demonstrated for the first time that altered methylation of gene promoters induced in the $F_{1}$ generation by protein restriction during fetal development can be inherited by the $\mathrm{F}_{2}$ generation, in the absence of the nutritional cues that induced epigenetic changes in the $F_{1}$.

In a subsequent paper [8] the authors aimed at clarifying the mechanism responsible for the observed hypomethylation at the GR $1_{10}$ promoter. Offspring of dams fed a PR diet were killed at postnatal day 34 and expression, methylation and recruitment at the GR $1_{10}$ promoter of enzymes involved in DNA methylation were analyzed by RT-PCR, methylation-sensitive PCR and chromatin immunoprecipitation assays. Methylation of the promoter for hepatic GR $1_{10}$ was reduced by $33 \%$ and expression of the GR $1_{10}$ gene was increased by $84 \%$ in the offspring of dams in the PR group, confirming some of the previous results. In addition, expression of the mRNA for DNA methyltransferase 1 (Dnmt1), which is responsible for the maintenance of methylations during cell division, and Dnmt 1 binding at the GR $1_{10}$ promoter were both lower in PR versus control offsprings (17 and 12\%, respectively). Conversely, mRNA expression of the other enzymes was similar in PR and control offsprings. Based on these data, the authors concluded that hypomethylation at the GR $1_{10}$ promoter was due to reduced capacity of Dnmt 1 to methylate DNA during mitosis and that it was gene-specific. When pregnant rats were fed a PR diet with a 5-fold excess of folic acid (provider of methyl groups) supplementation prevented the previously observed reduction in the expression of Dnmt1, stressing the role of methyl donors in the reversal of epigenetic effects caused by protein undernutrition during fetal life. The relevance of this mechanism to humans was tested by analyzing Dnmt 1 expression and GR1 promoter methylation in umbilical cord samples from 15 term infants of birth weight within normal range. Variation between the highest and the lowest value of GR1 promoter methylation was about 2-fold, showing for the first time that there is a considerable epigenetic variation of this gene within normal human population [8]. The expression of Dnmt1 was also linearly correlated with methylation of the GR1 promoter $(r=0.70)$ and the variations in the Dnmt1 expression were estimated to account for $49 \%$ of the observed variation in GR1 methylation. Thus, the authors proposed Dnmt1 expression as a key mechanism in the induction of different phenotypes in humans in response to varied prenatal conditions.

A subsequent paper [5] focused on developmental plasticity, the mechanism by which epigenetic changes are directionally modulated by time and order of exposure to different cues to produce distinct phenotypes. To introduce the first nutritional "cue" to developmental plasticity, rats were fed a standard diet throughout gestation, having access to either $30 \%$ the amount (UN undernutrition group) or eating ad libitum, (AD group). After birth, female pups from $\mathrm{UN}$ and $\mathrm{AD}$ mothers were fostered to $\mathrm{AD}$ mothers. From post-natal day 3, randomized groups of pups from both $\mathrm{UN}$ and $\mathrm{AD}$ received either leptin or saline for 10 days as a second nutrition-related cue, while freely eating a standard diet. Leptin was postulated to act as adiposity signal even in the absence of excess adipose tissue. At weaning, all the groups (leptin-treated and salinetreated rats from either $\mathrm{AD}$ or $\mathrm{UN}$ mothers) were placed on either standard chow (control) or high fat diet as a third nutritional cue. Methylation of gluocorticoid receptor (GR) and PPAR $\alpha$ promoters and expression of GR, PPAR $\alpha$, PEPCK and $11 \beta$-hydroxysteroid dehydrogenase type 2 (11 $\beta$-HSD2), the enzyme that inactivates corticosteroids, were studied in the liver of adult rats from all groups. The main result was that post-natal leptin exposure showed opposite effects, depending on previous in utero nutrition. Methylation of both promoters was elevated by leptin in AD groups but was decreased (for PPAR $\alpha$ ) or unaffected (for GR) in UN groups. Interactions between prenatal nutrition and leptin administration were significant for both PPAR $\alpha$ and GR methylation. Leptin administration also increased $11 \beta$-HSD2 gene expression in AD groups but decreased it in UN groups. The bidirectional effects of leptin were independent of post-weaning nutrition (normal chow or high fat diet). The authors speculated that prenatal nutrition (first cue) induced a bifurcation in the effects of the second cue (leptin administration) on gene expression and methylation.

To conclude, I would like to present the "mismatch hypothesis" as discussed in Godfrey et al. [6]. Phenotype induction is described as the attempt to match an individual's response to a predicted environment, a way to improve fitness of the species in a changing environment. Adjustment to the foreseen postnatal environment is made during fetal life by epigenetic changes in response to maternal environmental cues. However, the epigenetic response can "mismatch" the environment experienced throughout life. This can happen when nutritional cues from the mother are inaccurate, due to imbalanced or insufficient diet during pregnancy, inappropriate placental transport and/or a rapidly changing postnatal environment. Examples in humans are, for instance, emigration to a country with very different nutritional habits, improved socioeconomic conditions, or rapid economic transitions in developing countries. Such mismatch between the information received during fetal life and the environment experienced postnatally can induce metabolic "unfit" phenotypes with increasing risk of developing pathological conditions like type-2 diabetes, 
obesity and cardiovascular disease. The non-genomic transmission of environmental information, implemented by epigenetics, evolved to improve the chances of the offsprings to survive and become efficient reproducers in their youth. Since, at least in developed countries, the average age of the population is far higher than that of our ancestors, this evolutionary mechanism may no longer be advantageous for us. The challenge of epigenetic research in the near future is to devise and implement epigenetic-driven nutritional intervention therapies to contrast the onset of such diseases from infancy to old age.

However, many questions are still open and I would like to suggest just a few of them:

1. Some target genes subject to epigenetic nutritional misregulation during fetal development have already been identified. Will this misregulation be restricted to only one subset of genes? And if so, which genes are the best candidates for epigenetic nutritional regulation during differentiation? McArdle et al. [10] observed that the phenotype arising from different nutritional stresses (i.e. Fe-deficient or low protein diets) is similar, irrespective of the pre-natal nutrient stressor. This finding has led to the concept of "gatekeeper genes" or "gatekeeper pathways", to indicate crucial points or pathways during development where diverse stresses can produce the same phenotypic outcome.

2. Are epigenetic changes mainly activating/silencing gatekeeper pathways?

3. Are there reasonable hopes of designing in the future "epigenetic-based intervention therapies" using "key molecules" as nutritional supplements (e.g. methyl donors or others)?

4. Since epigenetic changes are not exclusively restricted to fetal and neonatal life, how relevant could they be in maintaining good health during lifespan in combination with nutritional factors?

In conclusion, epigenetics is unravelling a continuous cross talk between our genetic profile and the environment. Indeed, genome is much more "flexible" than previously thought and such flexibility underscores the relevance of "good eating" for maintenance of good health. Future nutritionists will have to face the challenge of elucidating the mechanism of nutrition-induced epigenetic changes during pre and post-natal life, to optimize nutritional interventions in a "personalized" perspective.

\section{References}

1. Burdge G, Slater-Jefferies J, Torrens C, Phillips E, Hanson M, Lillycrop K (2007) Dietary protein restriction of pregnant rats in the $F_{0}$ generation induces altered methylation of hepatic genes promoters in the adult male offsprings in the $F_{1}$ and $F_{2}$ generations. Br J Nutr 97:453-439. doi: 10.1017/S0007114507352392

2. Cooney C (2007) Epigenetics-DNA-based mirror of our environment? Dis Markers 23:121-137

3. Fraga M, Ballestar E, Paz M,Ropero S, Setien F, Ballestar M, Heine Suner D, Cigudosa J, Urioste M, Beitez J, Boix-Chornet M, Sanchez-Aguilera A, Ling C, Carlsson E, Poulse P, Vaag A, Stephan Z, Spector T, Wu Y-Z, Plass C, Esteller M (2005) Epigenetic differences arise during the lifetime of monozygotic twins. Proc Natl Acad Sci USA 102:10604-10609. doi/10.1073/pnas.0500398102

4. Gluckman P, Hanson M (2004) The developmental origin of the metabolic syndrome. Trends Endocrinol Metab 15:183-187

5. Gluckman P, Lillycrop K, Vickers M, Pleasants A, Phillis E, Beedle A, Burdge G, Hanson M (2007) Metabolic plasticity during mammalian development is directionally dependent on early nutritional status. Proc Natl Acad Sci USA 104:1279612800. doi/10.1073/pnas.0705667104

6. Godfrey KM, Lillycrop KA, Burge GC, Gluckman PD, Hanson MA (2007) Epigenetic mechanism and the mismatch concept of the developmetal origins of health and disease. Pediatr Res 61:5R-10R. doi: 10.1203/pdr.0b013e318045bedb

7. Goldberg A, Allis C, Bernsterin E (2007) Epigenetics: a landscape takes shape. Cell 128:635-638. doi: 10.1016/j.cell.2007.02.006

8. Lillycrop K, Slater-Jeffries J, Hanson M, Godfrey K, Jackson A, Burdge $G$ (2007) Induction of altered epigenetic regulation of the hepatic glucocorticoid receptor in the offspring of rats fed a protein restricted diet during pregnancy suggest that reduced DNA methyltransferease-1 expression is involved in impaired DNA methylation and changes in histone modifications. Br J Nutr 97:1064-1073. doi: 10.1017/S0007114550769196X

9. Reamon-Buettner S, Borlak J (2007) A new paradigm in toxicology and teratology: altering gene activity in the absence of DNA sequence variation. Reprod Toxicol 24:20-30. doi: 10.1016/j.reprotox.2007.05.002

10. Richmond A, Gambling L, Mayer C, McArdle HJ (2007) The gatekeepers genes hypothesis: identification of gatekeepers genes in nutritional stress using DNA arrays. NuGO, Fourth European Nutrigenomic Conference, 18-21 September 2007, Oslo

11. Waddington C (1942) The epigenotype. Endeavour 1:18-20 